\title{
Environment and susceptibility of disulfide bonds towards radiation damage
}

Jesmita Dhar ${ }^{1}$, Rajasri Bhattacharyya ${ }^{1}$, Shubhra Ghosh Dastidar ${ }^{1}$, Pinak Chakrabarti ${ }^{1}$, Manfred S. Weiss ${ }^{2}$

${ }^{1}$ Bioinformatics Centre, Bose Institute, Kolkata, India, ${ }^{2}$ Helmholtz-Zentrum Berlin für Materialien und Energie, Macromolecular Crystallography (BESSY-MX), Albert-Einstein-Str., Berlin, Germany

E-mail: dhar.beauty@gmail.com

Radiation induced damage to protein crystals during data collection is a major impediment for obtaining accurate structural information on macromolecules. Some of the specific impairments inflicted upon highly brilliant X-ray irradiation are disulfide bond cleavage and the loss of the integrity of carboxyl groups of acidic residues. There are results to indicate that not all disulfide bridges are equally susceptible to damage. Consideration of the disulfide bonds in the structures of elastase, lysozyme and acetylcholinesterase indicate that the S-S bonds which have a close contact with a carbonyl oxygen atom along the extension of the bond are more susceptible to breakage. Such an arrangement predisposes electron transfer to occur from the oxygen atom to the disulfide bond leading to its reduction. The energetics and charge transfer in $\mathrm{S} \cdots \mathrm{O}$ interactions were quantified based on ab initio calculations, using 6-31G(d,p)++ basis set on models generated mimicking protein structures. The charge transfer not only depends on distance but also on geometric orientation of carbonyl $\mathrm{O}$ atom with respect to the $S$ atom involved in disulphide bond. Interaction between a nucleophile and electrophile, akin to hydrogen bonding, stabilizes protein structures; but this also provides a pathway of electron transfer to the S-S bond leading to its reduction during exposure of the protein crystal to intense X-ray beam.

Keywords: disulfide bond, radiation damage, $S \cdots O$ interaction 